\title{
ENHANCED ENDOSCOPY FOR THE DIAGNOSIS OF GASTRICANTRALINTESTINAL METAPLASIA
}

By

\author{
Mohammed Abd El-Rahiem Ahmed*, Magdy Abd El-Kariem El- \\ Dahshan*, Zakarya Mohammed Zakarya*and Shadia Hussien \\ Mabrouk** \\ *Department of Internal Medicine, Faculty of Medicine, Al-Azhar University \\ **Department of Clinical Pathology, Ain Shams University \\ Corresponding author: Mohammed Abd El-Rahiem Ahmed \\ E-mail: abdelrahiemamr6@gmail.com
}

\begin{abstract}
Background: The detection of early gastric cancer may improve survival and avoid major gastric surgeries. Gastric Intestinal Metaplasia (GIM) is a precancerous lesion of the stomach. Screening for GIM may therefore lead to early detection of gastric neoplastic lesions.

Objective: This study was designed to determine the effect of conventional Narrow Band Image (NBI) in combination with acetic acid (AA-NBI) on the diagnosis of antral GIM.

Patients and Methods: The present study was performed in 100 consecutive adults who required endoscopic examination at AL Hussein University Hospital, Department of Internal Medicine, Endoscopy Unit, Conventional white-light endoscopy (WLE), NBI and AA-NBI was performed in all patients by the same endoscopist during a single procedure with a GIF Q290 endoscope (Olympus Medical Systems, Tokyo, Japan), All suspicious antral gastric lesions was photographed. Any abnormal mucosal change, such as localized discoloration and rough areas, was considered indicative of GIM lesions.

Results: Fifty-six (56.0\%) out of the 100 individuals examined were found to have GIM (by biopsy), of which $34(60.7 \%)$ were diagnosed correctly by NBI (sensitivity $66.7 \%$ and specificity $68.2 \%$ ) and $42(75 \%)$ were correctly identified by AA-NBI (sensitivity 75\% and specificity 73.91\%), as compared to only 24 $(42.86 \%$ ) by WLE (sensitivity $42.86 \%$ and specificity $37.5 \%$ ). Therefore, the sensitivity of AA-NBI in the diagnosis of GIM was significantly higher than NBI $(\mathrm{p}<0.05)$ and WLE $(\mathrm{p}<0.001)$. Our study indicates that AA-NBI can improve the accuracy of endoscopy-targeted biopsies for GIM.

Conclusion: Acetic acid staining combined with NBI was of a higher sensitivity and specificity in the diagnosis of intestinal metaplasia in gastric mucosa, which was in good consistency with the pathological diagnosis. Compared with the normal NBI, it was more capable to detect lesions of intestinal metaplasia in gastric mucosa, improved the accuracy of targeted biopsy to intestinal metaplasia, and has an important significance in the early detection and treatment of tumors.
\end{abstract}

Key words: Acetic acid, NBI, GIM, Antrum.

\section{INTRODUCTION}

Gastric cancer is the third leading cause of cancer death worldwide (Focket al., 2014). Gastric cancer onset is considered a multistep process that includes the consecutive development of chronic gastritis followed by mucosal atrophy, gastric intestinal metaplasia (GIM), dysplasia, and finally adenocarcinoma. The surveillance of 
patients with GIM may therefore lead to the earlier detection of advanced precancerous lesions and gastric cancer.

The gold standard for diagnosing GIM remains the histology of biopsy specimens. However, the major limitation of this approach is that GIM exhibits few macroscopic morphological changes, and as a consequence, GIM may readily be missed with random biopsy sampling. Recently, several new endoscopic techniques have been developed to increase the detection of GIM, including chromoendoscopy, auto fluorescence imaging, confocal laser endomicroscopy flexible spectral imaging color enhancement, narrow band imaging (NBI) and magnification endoscopy, (Lieta., 2013 and So et al., 2013).

Currently, there is still no unified standard for chromoendoscopy in the diagnosis of GIM. Moreover, the use of methylene blue carries the risk of causing oxidative DNA damage (Hardieet al., 2012), while auto fluorescence imaging, confocal laser endomicroscopy and flexible spectral imaging color enhancement are hard to manipulate. Therefore, these techniques are not generally used in clinical practice.

NBI is an endoscopic imaging technology, which results in the good contrast of surface structures and vascular architecture in the superficial mucosa using blue (400-430 $\mathrm{nm}$ ) and green (535$565 \mathrm{~nm})$ narrow-band light. These wavelengths are close to the light absorption peaks of hemoglobin (An et al., 2012). NBI with magnification endoscopy can provide a microscopic image of the mucosal and vascular structures, which are used for the detection of GIM (An et al., 2012 and Savarino et al., 2013). Acetic acid is a weak acid that breaks the disulfide bonds of glycoproteins of the mucus layer, causing reversible denaturation of the intracellular cytoplasmic proteins. In the columnar epithelium, acetic acid leads to the enhancement of the mucosal architecture and pit-pattern (Tao et al., 2014). Acetic acid combined with magnification endoscopy or indigo carmine has been used to diagnose gastric neoplasia Lee et al., 2010 (Konoet al., 2014, and Tao et al., 2014).

The present work aimed to study the effect of NBI in combination with acetic acid (AA-NBI) in the diagnosis of GIM.

\section{PATIENTS AND METHODS}

The present study was performed on 100 consecutive adults who required endoscopic examination at $\mathrm{Al}$ Hussein University Hospital, Endoscopy Unit Department of Internal Medicine. Written informed consent was obtained from all patients before examination. All procedure followed Al-Azhar University research committee regulations. The study was carried out during the period from December2017 to July, 2018.

\section{Exclusion criteria:}

Patients with advanced gastric cancer, previous gastrectomy or partial gastric resection, on-going treatment with antiplatelet medication, anticoagulant medication or no steroidal antiinflammatory drugs, and the presence of hemorrhagic diseases.

\section{Endoscopic Procedures:}

Conventional white-light endoscopy (WLE), NBI and AA-NBI were performed 
in all patients by the same endoscopist during a single procedure with a GIF Q290 endoscope (Olympus Medical Systems, Tokyo, Japan). Because the gastric antrum one of the regions with the highest prevalence of GIM and the acetic acid whitening time was only a few seconds to a few minutes. It is difficult to observe the entire stomach during such a short time. Therefore, we selected the gastric antrum as the regions for examination in this study. Mucus adhering to the mucosa of the gastric antrum was washed away as thoroughly as possible. All suspicious antral gastric lesions were photographed. Any abnormal mucosal change, such as localized discoloration and rough areas, were considered indicative of GIM lesions. The NBI system was used to carefully observe the gastric antrum. NBI suspicious lesions for GIM were defined as bluish-whitish areas with a regular mucosal pattern. Finally, acetic acid diluted with water $(2 \%)$ was applied to the antrum in the NBI model. The positions of the lesions detected by WLE, NBI or AA-NBI were recorded to ensure the precision of the biopsies obtained. At least, one targeted biopsy was separately collected from the endoscopic lesions suspicious of GIM by AA-NBI or WLE, and two random biopsies were collect from the antrum in areas where there were no abnormal findings to serve as controls. If no suspected lesions were identified by AANBI, NBI and WLE, two random biopsies were taken from the antrum according to the updated Sydney classification (Dixon et al., 1996).

\section{Histopathology:}

All biopsy specimens were fixed in $4 \%$ formalin and embedded in paraffin. The slides were routinely processed with hematoxylin and eosin (H\&E) stains. The histological diagnosis will be reported according to the updated Sydney Classification for chronic gastritis and the modified Vienna criteria for neoplasia.

\section{Statistical Analysis:}

Data were analyzed using Statistical Program for Social Science (SPSS) version 24.For the per-patient analysis, the endoscopically suspected lesions in one patient were considered one unit of analysis in this evaluation. The sensitivity, specificity, positive predictive values, negative predictive values and accuracy for the prediction of GIM in the AA-NBI, NBI and WLE models were calculated using histology as a reference value. For the per-biopsy analysis, the diagnostic accuracy of the targeted biopsies for GIM for the AA-NBI and WLE models were calculated in each specimen. The chisquared test was used to statistically compare the two groups.

P-value $<0.05$ was considered significant insignificant.

\section{RESULTS}

A total of 100 eligible patients at AlHussein University Hospital were recruited for this study. Among 50 patients with GIM by AA-NBI, the mean age was $40.16 \pm 14.6$ years with minimum age of 18 years and maximum age of 68 years. 18 patients $(36 \%)$ were males, while 32 patients $(64 \%)$ were females; 38 
patients $(76 \%)$ were non-smoker, while 12 patients (24\%) were smokers; All patients $(100 \%)$ were non-alcoholic, 36 patients $(72 \%)$ were non-diabetics, while 14 patients $(28 \%)$ were diabetics. The mean Body Mas Index (BMI) was $25.4 \pm 4.5$ with minimum BMI of 16 and maximum BMI of 34.3.As regard to $\mathrm{H}$ pylori $\mathrm{Ag}, 8$ patients $(16 \%)$ were negative, while 42 patients $(84 \%)$ were positive. As regard to HVC Ab, 48 patients $(96 \%)$ were negative, while 2 patients $(4 \%)$ were positive. All studied patients $(100 \%)$ were negative as regard HBS Ag, epigastric pain was recorded in 30 patients, GERD was recorded in 2 patients, dysphagia was recorded in 4 patients, recurrent vomiting was recorded in 4 patients, chronic diarrhea was recorded in 6 patients. Chronic cough was recorded in 4 patients; iron deficiency anemia was recorded in 2 patients. While weight loss and Iron deficiency anemia (IDA) was recorded in 4 patients (Table $\mathbf{1})$.

Table (1): Demographic data of patients with GIM by AA-NBI

\begin{tabular}{|c|c|c|}
\hline & & $\begin{array}{c}\text { Studied patients } \\
(\mathrm{N}=100)\end{array}$ \\
\hline \multirow{2}{*}{ Age } & Mean \pm SD & $40.16 \pm 14.6$ \\
\hline & Min - Max & $18-68$ \\
\hline \multirow{2}{*}{ Sex } & Male & $18(36 \%)$ \\
\hline & Female & $32(64 \%)$ \\
\hline \multirow{2}{*}{ Smoking } & No & $38(76 \%)$ \\
\hline & Yes & $12(24 \%)$ \\
\hline \multirow{2}{*}{ Alcohol } & No & $50(100 \%)$ \\
\hline & Yes & $0(0 \%)$ \\
\hline \multirow{2}{*}{$\mathrm{DM}$} & No & $36(72 \%)$ \\
\hline & Yes & $14(28 \%)$ \\
\hline \multirow{2}{*}{ BMI } & Mean \pm SD & $25.4 \pm 4.5$ \\
\hline & Min - Max & $16-34.3$ \\
\hline \multirow{2}{*}{$\mathrm{H}$ pylori $\mathrm{Ag}$} & Negative & $8(16 \%)$ \\
\hline & Positive & $42(84 \%)$ \\
\hline \multirow{2}{*}{$\mathrm{HCV} \mathrm{Ab}$} & Negative & $48(96 \%)$ \\
\hline & Positive & $2(4 \%)$ \\
\hline $\mathrm{HBs} \mathrm{Ag}$ & Negative & $50(100 \%)$ \\
\hline \multirow{2}{*}{ Drug abuse } & No & $32(64 \%)$ \\
\hline & Yes & $18(36 \%)$ \\
\hline \multirow{2}{*}{ Associated morbidity } & No & $42(84 \%)$ \\
\hline & Yes & $8(16 \%)$ \\
\hline \multirow{10}{*}{ Indications of endoscope } & Epigastric pain & 30 \\
\hline & GERD & 2 \\
\hline & Hematemesis & $\mathbf{0}$ \\
\hline & Dysphagia & 4 \\
\hline & Recurrent vomiting & 4 \\
\hline & Chronic diarrhea & 6 \\
\hline & atypical chest pain & $\mathbf{0}$ \\
\hline & Chronic cough & 4 \\
\hline & Iron deficiency anemia & 2 \\
\hline & weight loss, IDA & 4 \\
\hline
\end{tabular}


Among 44 patients with positive GIM by NBI, there were 12 patients $(27.3 \%)$ showed bluish patch, 4 patients $(9.1 \%)$ showed light blue crest, 18 patients $(40.9 \%)$ showed villous and 10 patients (22.7\%) showed white opaque substance.
As regard AA-NBI, there were 12 out of 50 patients $(24 \%)$ showed bluish patch, 4 patients $(8 \%)$ showed light blue crest, 24 patients $(48 \%)$ showed villous and 10 patients $(20 \%)$ showed white opaque substance (Table 2).

Table (2): Description of gastric metaplasia morphology by the NBI and AA-NBI

\begin{tabular}{|c|c|c|c|c|}
\hline \multirow{2}{*}{$\begin{array}{l}\text { Gastric } \\
\text { metaplasia morphology }\end{array}$} & \multicolumn{2}{|c|}{$\begin{array}{c}\text { NBI } \\
(\mathbf{n = 4 4})\end{array}$} & \multicolumn{2}{c|}{$\begin{array}{c}\text { AA-NBI } \\
(\mathbf{n}=\mathbf{5 0})\end{array}$} \\
\hline Bluish patch & 12 & $27.3 \%$ & 12 & $24 \%$ \\
\hline Light blue crest & 4 & $9.1 \%$ & 4 & $8 \%$ \\
\hline Villous & 18 & $40.9 \%$ & 24 & $48 \%$ \\
\hline White opaque substance & 10 & $22.7 \%$ & 10 & $20 \%$ \\
\hline
\end{tabular}

Among 30 patients with positive GIM by WLI there were 18 patients $(60 \%)$ showed whitish area, 10 patients $(33.3 \%)$ showed reddish area and 2 patients $(6.7 \%)$ showed rough area. As regard AA-WLI, there were 20 patients out of $36(55.6 \%)$ showed whitish area, 12 patients $(33.3 \%)$ showed reddish area and 4 patients $(11.1 \%)$ showed rough area (Table 3).

Table (3): Description of gastric metaplasia morphology by the WLI and AA-WLI

\begin{tabular}{|c|c|c|c|c|}
\hline \multirow{2}{*}{$\begin{array}{l}\text { Gastric } \\
\text { metaplasia morphology }\end{array}$} & \multicolumn{2}{|c|}{$\begin{array}{c}\text { WLI } \\
(\mathbf{n = 3 0})\end{array}$} & \multicolumn{2}{c|}{$\begin{array}{c}\text { AA-WLI } \\
(\mathbf{n = 3 6})\end{array}$} \\
\hline Whitish area & 18 & $60 \%$ & 20 & $55.6 \%$ \\
\hline Reddish area & 10 & $33.3 \%$ & 12 & $33.3 \%$ \\
\hline Rough area & 2 & $6.7 \%$ & 4 & $11.1 \%$ \\
\hline
\end{tabular}

The diagnostic performance of studied endoscopes in relation to Histopathology results showed that the WLI showed sensitivity of $42.86 \%$, specificity of $37.5 \%$, PPV of $28.57 \%$, NPV of $52.94 \%$ and accuracy of $39.17 \%$ in diagnosis of GIM, the NBI showed sensitivity of $64.29 \%$, specificity of $62.96 \%$, PPV of $64.29 \%$, NPV of $62.56 \%$ and accuracy of $63.64 \%$ in diagnosis of GIM. The AAWLI showed sensitivity of $50 \%$, specificity of $37.37 \%$, PPV of $41.18 \%$, NPV of $56.25 \%$ and accuracy of $48.48 \%$ in diagnosis of GIM. The AA-NBI showed sensitivity of $75 \%$, specificity of $73.91 \%$, PPV of $80 \%$, NPV of $68 \%$ and accuracy of $74.55 \%$ in diagnosis of GIM, the WLI + NBI showed the sensitivity of $57.43 \%$, specificity of $48.65 \%$, PPV of $48.65 \%$, NPV of $51.43 \%$ and accuracy of $50 \%$ in diagnosis of GIM, the WLI+AAWLI showed sensitivity of $46.43 \%$, specificity of $43.18 \%$, PPV of $34.21 \%$, NPV of $55.88 \%$ and accuracy of $44.44 \%$ in diagnosis of GIM, the NBI + AA-NBI showed sensitivity of $75.76 \%$, specificity of $77.27 \%$, PPV of $83.33 \%$, NPV of $68 \%$ and accuracy of $76.36 \%$ in diagnosis of $\mathrm{GIM}$, the WLI + BNI + AA-WLI + AANBI showed sensitivity of $80 \%$, specificity of $78.26 \%$, PPV of $80 \%$, NPV of $78.26 \%$ and accuracy of $79.14 \%$ in diagnosis of GIM (Table 4). 
Table (4): Diagnostic performance of studied endoscopes in relation to Histopathology results

\begin{tabular}{|c|c|c|c|c|c|}
\hline Parameter & Sensitivity & Specificity & PPV & NPV & Accuracy \\
\hline WLI & $42.86 \%$ & $37.5 \%$ & $28.57 \%$ & $52.94 \%$ & $39.17 \%$ \\
\hline NBI & $64.29 \%$ & $62.96 \%$ & $64.29 \%$ & $62.56 \%$ & $63.64 \%$ \\
\hline AA-WLI & $50 \%$ & $37.37 \%$ & $41.18 \%$ & $56.25 \%$ & $48.48 \%$ \\
\hline AA-NBI & $75 \%$ & $73.91 \%$ & $80 \%$ & $68 \%$ & $74.55 \%$ \\
\hline WLI + NBI & $57.43 \%$ & $48.65 \%$ & $48.65 \%$ & $51.43 \%$ & $50 \%$ \\
\hline WLI + AA-WLI & $46.43 \%$ & $43.18 \%$ & $34.21 \%$ & $55.88 \%$ & $44.44 \%$ \\
\hline NBI + AA-NBI & $75.76 \%$ & $77.27 \%$ & $83.33 \%$ & $68 \%$ & $76.36 \%$ \\
\hline $\begin{array}{c}\text { WLI + NBI +AA- } \\
\text { WLI + AA-NBI }\end{array}$ & $80 \%$ & $78.26 \%$ & $80 \%$ & $78.26 \%$ & $79.14 \%$ \\
\hline
\end{tabular}

Diagnostic Accuracy of Endoscopy for Patients with GIM (Per-Patient Analysis):

GIM primarily showed bluish-whitish areas and villous pattern in the NBI model (Figs 1B and 2B), and whitish patches in the AA-NBI model (Figs 1C, and 2C). neither patient with low-grade intraepithelial neoplasia Nor patients with high-grade intraepithelial neoplasia or gastric cancer were identified. Among all the patients examined, $56(56.0 \%)$ were histologically confirmed with GIM.
Diagnostic Accuracy of Targeted Biopsies for GIM (Per-biopsy Analysis):

For AA-NBI, a total of 137 targeted biopsies were obtained. Of these, 75 specimens were histologically diagnosed as GIM, and 62 were chronic inflammation. For WLE, a total of 145 targeted biopsies were obtained. Of these, 35specimens were diagnosed as GIM and 110 were chronic inflammation. Therefore, for the per- biopsy analysis, AA-NBI with targeted biopsies had a significantly greater diagnostic ability for GIM compared with WLE, with $54.74 \%$ $(75 / 137)$ versus $24.13 \% \quad(35 / 145)$ $(\mathrm{P}<0.001)$.
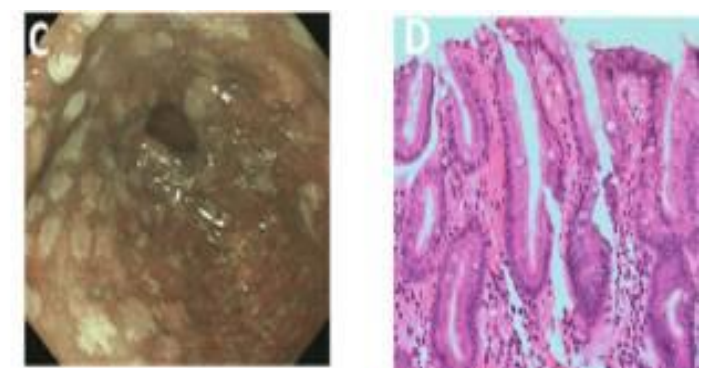

Figure (1): .Appearance of intestinal metaplasia in the antrum of the same patient under three different endoscopic models. A, Endoscopic image in WLE shows ash-colored nodular changes. B, After being switched to the NBI model, the lesions exhibit as bluish-whitish areas. $\mathrm{C}$, The clearer whitish patches are observed after sprinkling with acetic acid in the AA-NBI model. D, Targeted biopsy shows intestinal metaplasia of the stomach. 

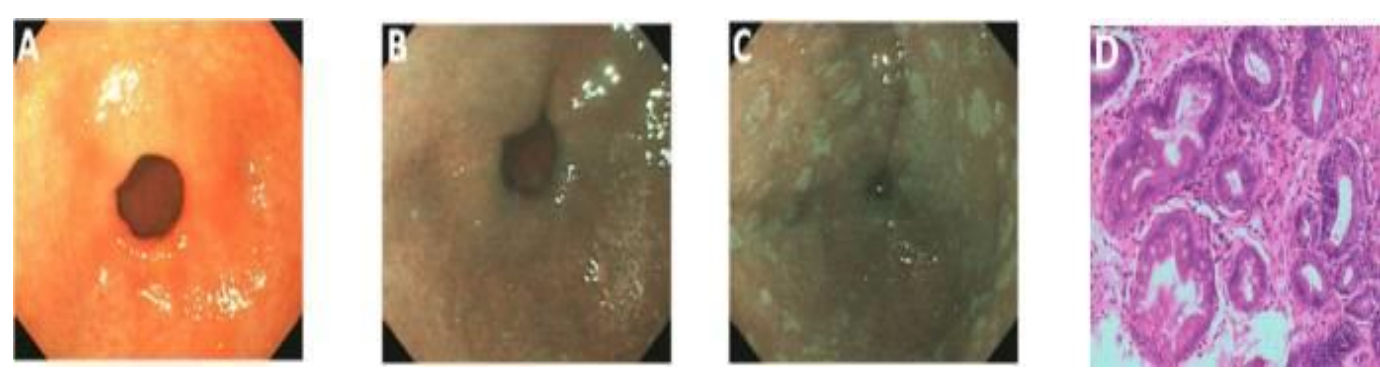

Figure (2): Appearance of intestinal metaplasia in the antrum of the same patient under three different endoscopic models. A, Endoscopic image in WLE shows antrum mucosa is normal. B, After being switched to the NBI model, bluish-whitish areas appear. $\mathrm{C}$, The clearer whitish patches are observed after sprinkling with acetic acid the AA-NBI model. D, Targeted biopsy shows intestinal metaplasia of the stomach.

\section{DISCUSSION}

The diagnosis of GIM using conventional WLE was unreliable because GIM usually appears in flat mucosa with few macroscopic morphological changes and occurs multifocal. Lim et al. (2013) defined GIM as the presence of whitish plaques, patches, or homogeneous whitish discoloration on the gastric mucosa and reported that the sensitivity and specificity were $24.0 \%$ and $91.9 \%$ for the lesions in the antrum respectively, and $24.2 \%$ and $88.0 \%$ for the lesions in the body, respectively. These results from various hospitals showed that the sensitivity of conventional WLE for the diagnosis of GIM is very low (Lim et al., 2013). Therefore, there is an urgency to increase the sensitivity of endoscopic diagnosis of GIM.

The NBI technique is based on a modification of the spectral characteristics of the optical filter in the light source, resulting in improved visibility of the mucosal structures (An et al., 2012). It is a unique sequential electronic endoscopy system. One of the greatest advantages of this system is its capacity of visualizing the minute mucosal surface without chromoendoscopy. However, magnifying endoscopes have not been widely used due to their complicated operation. Tao et al. (2014) has found that the results of ME-NBI for the diagnosis of early gastric cancer were the sensitivity, specificity, positive predictive value, negative predictive value, and accuracy of $87.2 \%$, $98.6 \%, 82.1 \%, \quad 99.0 \%$ and $97.8 \%$, respectively. The transient white coloration of the epithelial surface, which occurs after the spraying of acetic acid, is a phenomenon of increased opacity. This corresponds to the reversible alteration of the tertiary structures of cellular proteins upon applying the acetic acid for spraying the mucosa under the endoscope, whitening phenomenon appeared on the surface of the mucosa. So the observation of gastric mucosa would be more intuitive and stereoscopic; In addition, acetic acid can neutralize a small number of alkaline gastric mucus, to the extent that the microstructure of the mucosa would be displayed more clearly. Several reports also described acetic acid with indigo carmine for the diagnosis of early gastric cancer (Lee et al., 2010 and Kono et al., 2014). In our study, we firstly combined NBI with acetic acid (AA-NBI) for the 
diagnosis of GIM. We observed that ashcolored nodular lesions in WLE appear bluish-whitish in the NBI model alone, and whitish patches are observed after sprinkling acetic acid (AA-NBI).No ashcolored nodular changes were observed in WLE, and the lesions also appeared bluish- whitish in the NBI model. However, the clearer whitish patches were observed after sprinkling with acetic acid. The area showed no abnormal change in WLE and NBI, but become a whitish in the AA-NBI. Our study showed that AANBI allowed us to detect GIM in patients with a sensitivity of $87.9 \%$, a specificity of $68.2 \%$, a positive predictive value of $73.4 \%$, a negative predictive value of $84.9 \%$ and an accuracy of $78.0 \%$, all of which were significantly higher than those of WLE. Our study also showed that bluish-whitish areas were not found in the NBI model in some GIM subjects, whereas whitish patches appeared in the AA-NBI. Overall, compared to NBI, the sensitivity for the detection of GIM increased by $21.2 \%$ for AA-NBI, and the negative predictive value for the latter was also higher than that of NBI. The sensitivity, specificity, positive predictive value, and negative predictive value in our study using NBI are close to those published by Capelle et al. (2010) who achieved a sensitivity of $71 \%$, a specificity of $58 \%$, a positive predictive value of $65.0 \%$ and a negative predictive value of $65.0 \%$ using NBI for the diagnosis of GIM. The high sensitivity, specificity and accuracy of acetic acid enhanced NBI in detection of GIM previously reported by Sha et al. (2017) who found a sensitivity of $87.9 \%$, a specificity of $68.2 \%$, a positive predictive value of $73.4 \%$, a negative predictive value of $84.9 \%$ and an accuracy of $78.0 \%$, all of which were significantly higher than those of NBI (sensitivity $66.7 \%$ and specificity $68.2 \%$ ) and WLE(sensitivity $33.3 \%$ and specificity 28.8\%).Therefore, the sensitivity of AA-NBI in the diagnosis of GIM was significantly higher than NBI and WLE. Also, Meili et al. (2018) concluded that, In view of pathological results, the sensitivity of normal NBI for the diagnosis of intestinal metaplasia in gastric mucosa was $80.0 \%$, and the specificity was $83.3 \%$. The sensitivity of acetic acid staining combined with NBI for the diagnosis of intestinal metaplasia in gastric mucosa was $96.7 \%$, and the specificity was $100 \%$. The differences in sensitivity and specificity of the two groups were statistically significant. Our study indicated that AA-NBI can improve the accuracy of endoscopy-targeted biopsies for GIM. The advantage of this study was that NBI is a self-installed function of Gastroscopy System and easy to operate. White vinegar is a type of food, which is relatively easy to obtain, simple to prepare, and helpful to the clinic practice. Therefore, it is a good choice to utilize acetic acid staining combined with NBI to improve the detection rate of precancerous lesions in patients with mucosal abnormalities under the normal endoscopy. Our study had some limitations, as the study was performed at a single center only. Although it was necessary for our early stage comparison of the three relevant techniques. Obviously, larger multicenter prospective studies will be warranted to validate the findings from the current study. The endoscopic procedures for WLE and AANBI were performed by the same endoscopist for consistence. However, the 
detection of GIM using AA-NBI may cause bias because of the previous WLE observations. The antrum and angulus were selected in this study because they were the regions of the highest prevalence of intestinal metaplasia.

\section{CONCLUSION}

Acetic acid staining combined with NBI was of a higher sensitivity and specificity in the diagnosis of intestinal metaplasia in gastric mucosa, which was in good consistency with the pathological diagnosis. Compared with the normal NBI, it was more capable to detect lesions of intestinal metaplasia in gastric mucosa, improve the accuracy of targeted biopsy to intestinal metaplasia, and has an important significance in the early detection and treatment of tumors. In addition it was simple. This method was of good safety, economy and operability, and worthy of further clinical promotion.

\section{REFERENCES}

1. An JK, Song GA, Kim GH, Park do Y, Shin NR and Lee BE.(2012): Marginal turbid band and light blue crest, signs observed in magnifying narrow-band imaging endoscopy, are indicative of gastric intestinal metaplasia. BMC Gastroenterol., 12:169.

2. Capelle LG, Haringsma J, de Vries AC, Steyerberg EW, Biermann $K$ and van Dekken H. (2010): Narrow band imaging for the detection of gastric intestinal metaplasia and dysplasia during surveillance endoscopy. Dig Dis Sci., 55:3442-3448.

3. Fock KM. (2014): Review article: the epidemiology and prevention of gastric cancer. Aliment Pharmacol Ther; 40:250-260.

4. Kono Y, Takenaka R, Kawahara Y, Okada H, Hori $K$ and Kawano S. (2014): Chromoendoscopy of gastric adenoma using an acetic acid indigocarmine mixture. World $\mathrm{J}$ Gastroenterol; 20:5092-5097.
5. Lee BE, Kim GH, Park do Y, Kim DH, Jeon TY, Park SB and You HS. (2010): Acetic acid-indigo carmine chromoendoscopy for delineating early gastric cancers: its usefulness according to histological type. BMC Gastroenterol., 10:97-100.

6. Li Z, Zuo XL, Yu T, Gu XM, Zhou CJ and Li CQ. (2014): Confocal laser endomicroscopy for in vivo detection of gastric intestinal metaplasia: a randomized controlled trial. Endoscopy, 46:282-290.Lim JH, Kim N, Lee HS, Choe G, JoSY and Chon I(2013): Correlation between Endoscopic and Histological Diagnoses of Gastric Intestinal Metaplasia. Gut Liver; 7:41-50.

7. Meili G. Ji C and Hong C (2018): Diagnostic value of endoscopic acetic acid staining combined with narrow band imaging in the diagnosis of intestinal metaplasia in gastric mucosa Department of Gastroenterology, The Third Affiliated Hospital of Inner Mongolia Medical University, Baotou, Inner Mongolia, China36: 1657-1665.

8. Savarino E, Corbo $M$, Dulbecco $\mathbf{P}$, Gemignani L, Giambruno $E$ and Mastracci L. (2013): Narrow-band imaging with magnifying endoscopy is accurate for detecting gastric intestinal metaplasia. World J Gastroenterol., 19:2668-2675.

9. ShaJ Wang P, Zhu B, Minghui Z, Xueliang L and Feng G. (2017): Acetic Acid Enhanced Narrow Band Imaging for the diagnosis of Gastric Intestinal Metaplasia. PLoS ONE 12(1): e0170957.

10.So J, Rajnakova A, Chan YH, Tay A, Shah N, Salto and Tellez M. (2013): Endoscopic tri-modal imaging improves detection of gastric intestinal metaplasia among a high-risk patient population in Singapore. Dig Dis Sci., 58:3566-3575.

11. Tao G, Xing-Hua L, Ai-Ming Y, Wei-Xun Z, Fang $\mathrm{Y}$ and $\mathrm{Xi}$ W. (2014): Enhanced magnifying endoscopy for differential diagnosis of superficial gastric lesions identified with white-light endoscopy. Gastric Cancer., 17:122-129. 
دور المنظلر ضيق النطاق المدعوم بحمض الخليك في تشخيص حؤول الامعاء في غار المعدة

محمدعبدالرحيم أحمد*، مجدي عبدالكريم الدهانث*، زكريا محمد زكرياث، شادية * حسين مبروث

*قسم الامر اض الباطنة كلية الطب جامعة الازهر ،**قسم الباثولوجيا الاكلينيكية جامعة عين شمس

E-mail: abdelrahiemamr6@gmail.com

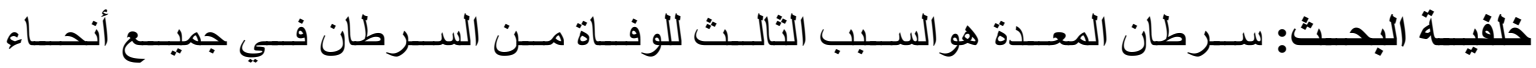

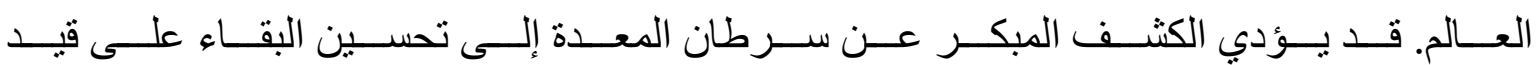

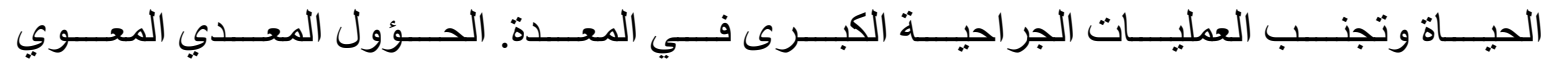

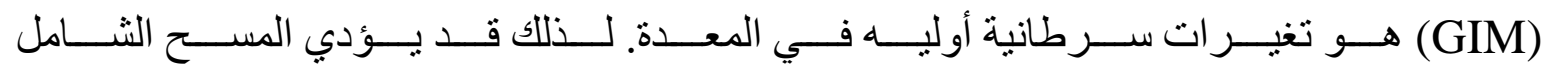
للحؤول المعدي المعوي إلى الكثف المبكر عن الأورام المعدية.

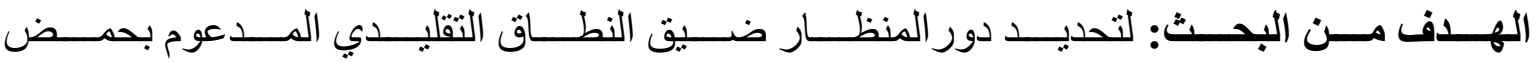

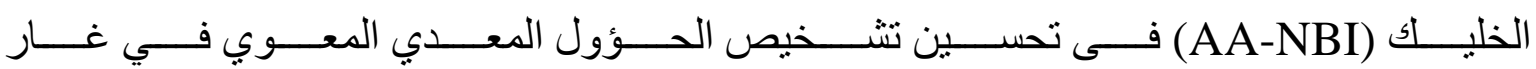

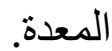

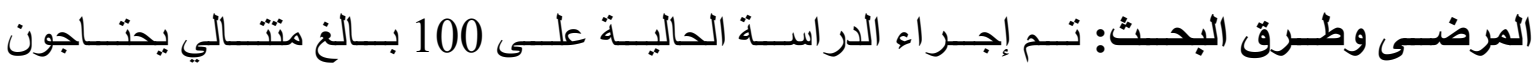

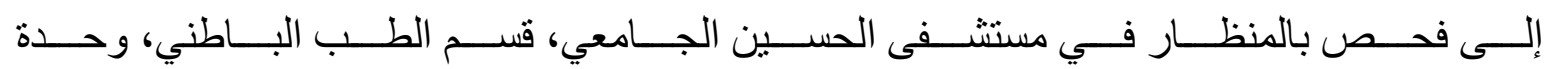

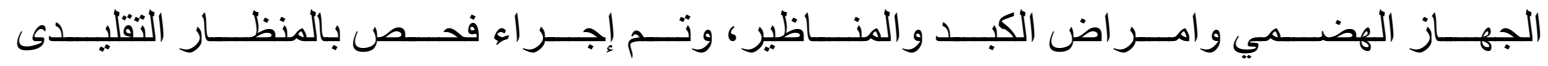

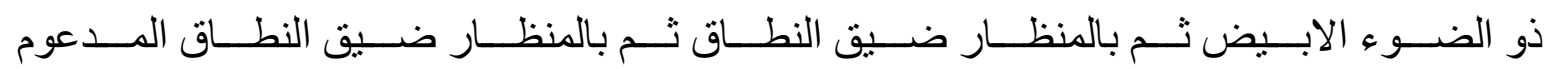

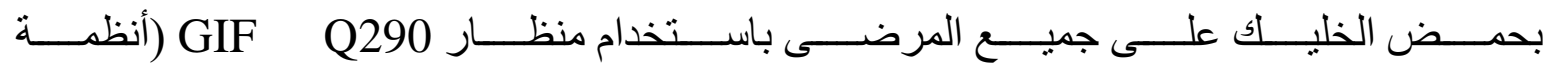

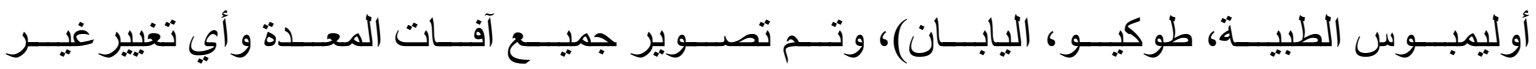

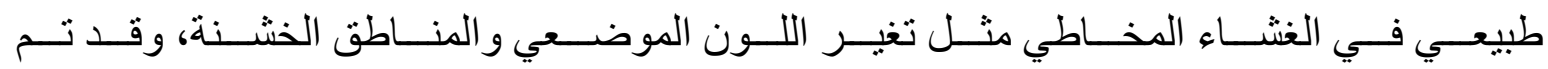

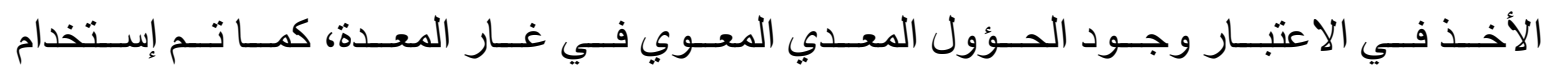

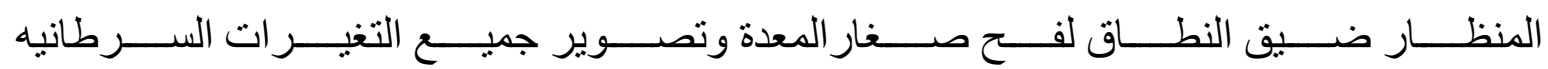
المشكوك بها.

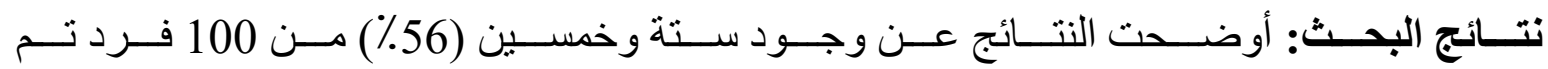

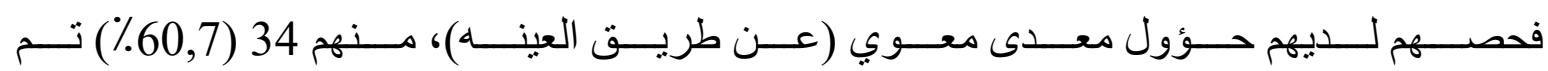




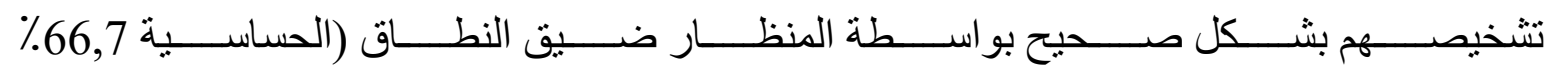

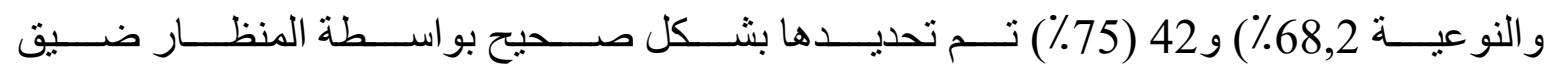

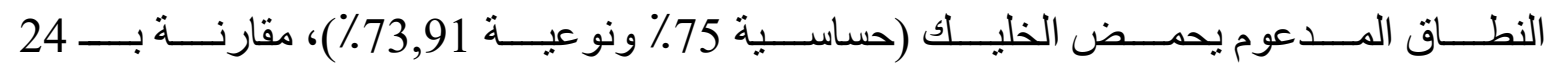

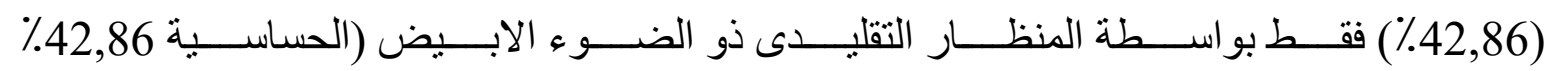

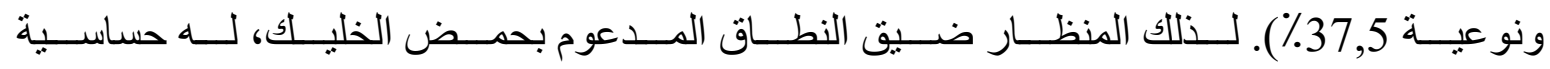

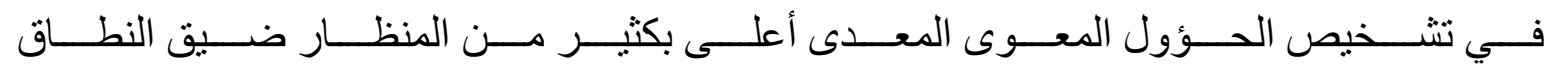
و المنظار التقليدي ذو الضوء الابيض.

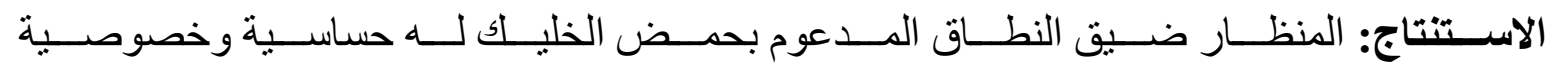

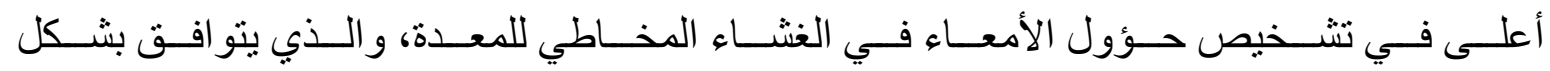

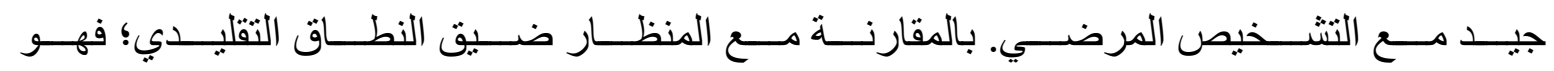

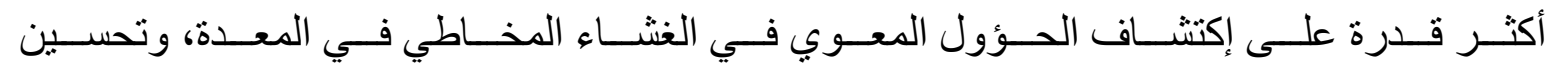

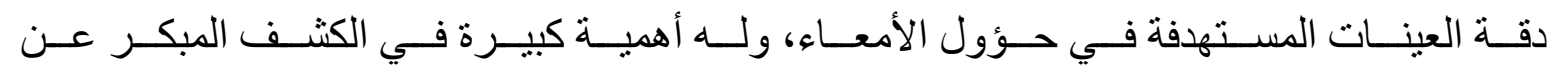
الأور ام و علاجها.

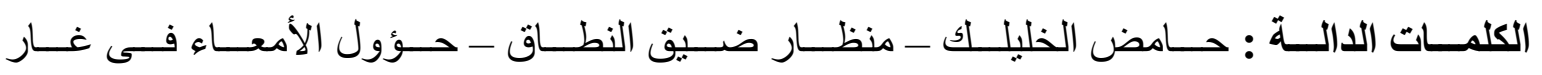
المعدة . 